\title{
76. Sur la classe projective d'un ensemble défini par l'induction transfinie.
}

Par Isamu NAKAHARA.

(Comm. by Z. Suetuna, M.J.A., July 12, 1952.)

En 1936 M. C. Kuratowski a démontré ${ }^{1)}$, que l'application de l'induction transfinie dans le domaine des ensembles projectifs ne conduit pas au dehors de ce domaine et évalué la classe projective d'un ensemble universel de ceux boreliens qui est donné par l'induction transfinie. Or, cette évaluation est amélioré en 1937 M. M. C. Kuratowski et J. v. Neumann ${ }^{2)}$. Ils ont donné un théorème fondamental sur l'induction transfinie et déterminé telle évaluation comme une application de lui. D'ailleur, elle n'est pas parfait et d'ou, nous pouvons l'amélirer encore. Le but de cette note est de donner telle évaluation amélioré.

1. Nous commençons par quelques définitions sur les notions et les notations que nous employons dans la suite.

Soit $\left\{r_{r}\right\}(n=1,2, \ldots)$ une suite bien déterminée et fixée des nombres rationnels contenus dans l'interval $<0,1\rangle$. Puis, pour un élément $x$ du discontinu $C$ de G. Cantor, nous désignons par $M_{x}$ l'ensemble des nombres rationnels $r_{n}$ tels qu'on ait $c_{n}=2$ dans la prolongation triadique de $x$ :

$$
x=\frac{c_{1}}{3}+\frac{c_{2}}{3^{2}}+\cdots+\frac{c_{n}}{3^{n}}+\cdots
$$

où $c_{n}=0$ ou bien 2 et par $\bar{x}$ le type de $M_{x}$ par rapport à l'ordre naturel.

Nous posons encore

$$
x_{(n)}=\frac{c_{1.2^{n}}}{3}+\frac{c_{3.2^{n}}}{3^{2}}+\cdots+\frac{c_{(2 t-1) 2^{n}}}{3^{i}}+\cdots \quad(i=0,1,2, \ldots)
$$

pour un élément $x$ de $C$ et désignons par $x^{(n)}$ un nombre réel tel que $M_{x}(n)$ se composé de tout $r_{i}$ qui remplit $r_{i}<r_{n}$ s'il $y$ a un nombre $r_{k}$ dans $M_{\boldsymbol{x}}$ tel que $r_{k} \geqq r_{n}$ et sinons, $x^{(n)}=0$. Puis, désignons par $C_{0}$ l'ensemble de $x$ de $C$ tel que $M_{x}$ soit bien ordonné.

Maintenant, en désignant par $A(y)$ une fonction universelle pour les ensembles ouvert et ceux fermés et dont le domaine est $\boldsymbol{C}$, nous définisons l'ensemble $L(x, y)$, où $x \in \boldsymbol{C}_{0}$ et $y \in \boldsymbol{C}_{0}$, par l'induction transfinie comme il suit,

$$
L(0, y)=A(y),
$$

1) C. R. Paris t. 202 (1936) et Fund. Math. t. 27 (1936).

2) Ann. of Math. Vol. 38 (1937). 
No. 7.] Sur la classe projective d'un ensemble défini par l'induction transfinie.

$$
L(x, y)=\limsup _{n \rightarrow \infty} L\left(x^{(n)}, y_{(n)}\right), \quad \text { si } \quad x \neq 0 .
$$

Alors, l'ensemble spatial

$$
Z=\underset{x, y, z}{E}[z \in L(x, y)]
$$

est ce qui est appellé l'ensemble de Lebesgue. Or, il et M. J. v. Neumann ont généralisé la définition de $Z$ comme il suit. Soient $f_{n}(x)$ et $g_{n}(y)(n=1,2, \ldots)$ les fonctions de Baire, définies sur $\boldsymbol{C}$ et $J^{3)}$ respectivement telles que $f_{n}(0)=0$ et $\overline{f_{n}(x)}<\bar{x}$ pour tout $x$ de $C_{0}$, et $\Phi_{B}$ un $\delta_{S}$-opération de F. H. Hausdorff dont la base est $B$. Alors, en prenant

$$
\begin{aligned}
& L(0, y)=A(y), \\
& L(x, y)=\sum_{s \in B} \prod_{n=1}^{\infty} L\left(f_{s_{n}}(x), g_{s_{n}}(y)\right), \quad \text { si } \quad x \neq 0,
\end{aligned}
$$

où $A(y)$ est une fonction universelle telle que $\underset{y, z}{E}[z \in A(y)]$ soit analytique, nous posons maintenant

$$
Z^{*}=\underset{x, y, z}{E}[z \in L(x, y)]
$$

2. Alors, ils ont demontré le

Théorème 1. Si $B$ est analytique dans $J, Z^{*}$ est aussi analytique par rapport à $\boldsymbol{C}_{0} \times \boldsymbol{J} \times \boldsymbol{J}$.

Or, en vertu de ce théorème, nous avons le

Théorème 2. L'ensemble $\boldsymbol{Z}$ est élémentaire par rapport à $\boldsymbol{C}_{0} \times \boldsymbol{C} \times \boldsymbol{J}$.

De plus, nous pouvons voir le

Théorème 3. Si $B$ est un ensemble $F_{\sigma}$ et si $\underset{y, z}{E[z \in A(y)]}$ est borelien, $Z^{*}$ est aussi élémentarie par rapport à $\boldsymbol{C}_{0} \times J \times J$.

3. Maintenant, nous démontrons le théorème 2. Si nous posons

nous avons

$$
\begin{aligned}
& L^{\prime}(0, y)=A^{c}(y)^{4)}, \\
& L^{\prime}\left(x^{(n)}, y_{(n)}\right)=L^{c}\left(x^{(n)}, y_{(n)}\right),
\end{aligned}
$$

$$
L^{\prime}(x, y)=\liminf _{n \rightarrow \infty} L^{c}\left(x^{(n)}, y_{(n)}\right),
$$

et de plus, $A^{c}(y)$ est une fonction universelle pour les ensembles ouverts et ceux fermés, dont le domaine est $G$. Nous avons donc

$$
\begin{aligned}
Z^{c} & =\operatorname{Comp} \cdot[\underset{x, y, z}{[}\{z \in L(x, y)\}]=\underset{x, y, z}{E}\left\{z \in L^{c}(x, y)\right\} \\
& =\underset{x, y, z}{E}\left\{z \in \operatorname{Comp} \cdot\left[\limsup _{n \rightarrow \infty} L\left(x^{(n)}, y_{(n)}\right)\right]\right\} \\
& =\underset{x, y, z}{E}\left\{z \in \liminf _{n \rightarrow \infty} L^{c}\left(x^{(n)}, y_{(n)}\right)\right\}=\underset{x, y, z}{E}\left\{z \in \liminf _{n \rightarrow \infty} L^{\prime}\left(x^{(n)}, y_{(n)}\right)\right\} \\
& =\underset{x, y, z}{E}\left\{z \in L^{\prime}(x, y)\right\}
\end{aligned}
$$

3) $\mathrm{J}$ est l'ensemble de nombres irrationneles dans l'intervale $\langle 0,1\rangle$.

4) Nous désignons par $\mathrm{A}^{c}$ le complémentaire de A. 
En autre part, $\liminf _{n \rightarrow \infty} E_{n}$ est une $\delta_{s}$-operation de F. Hausdorff dont la base est borelien et d'où, d'après le théorème $1, Z^{c}$ est analytique par rapport à $\boldsymbol{C}_{0} \times \boldsymbol{C} \times \boldsymbol{J}$.

$$
\text { c. } q \text {.f. d. }
$$

4. Puis, nous démontrons le théorème 3. D'après un théorème de M. M. L. Kantorovitch et E. Livenson ${ }^{5)}$, nous avons

$$
\tilde{B}^{c \boldsymbol{\theta})}=\mathrm{Comp} \operatorname{Proj}_{\mathcal{J}}\{(E \times J) . S\},
$$

où $S$ est un ensemble fermé dans $J \times J$ et donc, de même que le précédent, en posant

$$
\begin{aligned}
L^{\prime}(0, y) & =A^{c}(y) \\
L^{\prime}(x, y) & =\sum_{s \in B^{c}} \prod_{n=1}^{\infty} L^{c}\left(f_{s_{n}}(x), g_{s_{n}}(y)\right), \\
& =\sum_{S \in \widetilde{B}^{c}} \prod_{n=1}^{\infty} L^{c}\left(f_{s_{n}}(x), g_{s_{n}}(y)\right)
\end{aligned}
$$

où $x \in C_{0}$ et $y \in J$, nous avons

$$
Z^{* e}=\underset{x, y, z}{E}\left\{z \in L^{\prime}(x, y)\right\} \text {. }
$$

Par suite, en vertu du théorème $1, Z^{* c}$ est analytique par rapport à $\mathrm{C}_{0} \times J \times J$.

$$
\text { c. q. f. d. }
$$

5) L. Kantorovitch et E. Livenson: Memoire on the analytical operation and projective sets (I). F. M. t. 18 (1932) pp.

6) Pour la notation $\widetilde{\mathrm{B}}$, voir loc. cit. (6), p. 248. 